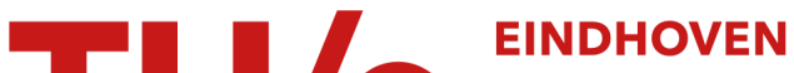 UNIVERSITY OF TECHNOLOGY
}

\section{Stability of networked control systems with large delays}

\section{Citation for published version (APA):}

Posthumus - Cloosterman, M. B. G., Wouw, van de, N., Heemels, W. P. M. H., \& Nijmeijer, H. (2007). Stability of networked control systems with large delays. In Proceedings of the 46th IEEE Conference on Decision and Control (CDC 46), 12-14 December 2007, New Orleans, USA (pp. 5017-5022). Institute of Electrical and Electronics Engineers. https://doi.org/10.1109/CDC.2007.4434669

DOI:

10.1109/CDC.2007.4434669

Document status and date:

Published: 01/01/2007

\section{Document Version:}

Publisher's PDF, also known as Version of Record (includes final page, issue and volume numbers)

\section{Please check the document version of this publication:}

- A submitted manuscript is the version of the article upon submission and before peer-review. There can be important differences between the submitted version and the official published version of record. People interested in the research are advised to contact the author for the final version of the publication, or visit the $\mathrm{DOI}$ to the publisher's website.

- The final author version and the galley proof are versions of the publication after peer review.

- The final published version features the final layout of the paper including the volume, issue and page numbers.

Link to publication

\section{General rights}

Copyright and moral rights for the publications made accessible in the public portal are retained by the authors and/or other copyright owners and it is a condition of accessing publications that users recognise and abide by the legal requirements associated with these rights.

- Users may download and print one copy of any publication from the public portal for the purpose of private study or research.

- You may not further distribute the material or use it for any profit-making activity or commercial gain

- You may freely distribute the URL identifying the publication in the public portal.

If the publication is distributed under the terms of Article 25fa of the Dutch Copyright Act, indicated by the "Taverne" license above, please follow below link for the End User Agreement:

www.tue.nl/taverne

Take down policy

If you believe that this document breaches copyright please contact us at:

openaccess@tue.nl

providing details and we will investigate your claim. 


\title{
Stability of Networked Control Systems with Large Delays
}

\author{
M.B.G. Cloosterman, N. van de Wouw, W.P.M.H. Heemels and H. Nijmeijer
}

\begin{abstract}
This paper proposes stability analysis techniques for Networked Control Systems (NCSs) with uncertain, timevarying network-induced delays. A discrete-time model, describing an NCS with delays, that can be both smaller and larger than the sampling interval, is presented. This model includes the effects of message rejection and vacancy sampling. The conditions are kept less conservative by exploiting the real Jordan form of the continuous-time model in the discretization. Based on this discrete-time NCS model sufficient LMI conditions are proposed for the stability analysis.
\end{abstract}

\section{INTRODUCTION}

Networked Control Systems (NCSs) are control systems in which (part of) the control loop is closed over a realtime network. The advantages of the use of an NCS are its flexible architecture [1] and a reduction of installation and maintenance costs [2]. The main disadvantages of NCSs are the network effects that influence the control loop, such as delays, variable sampling intervals and packet dropouts. Despite these disadvantages, NCSs are applied in a broad range of systems, e.g. mobile sensor networks, remote surgery, automated highway systems and unmanned aerial vehicles [1], [2]. In this paper the focus will be on the effects of delays on the stability of the control loop, where we restrict ourselves to systems without packet dropouts and with a constant sampling interval.

Most of the NCS literature on stability analysis deals with constant delays or time-varying delays smaller than the sampling interval. Here, we study NCS models including large delays, i.e. delays than can be both smaller and larger than the sampling interval. In [3], [4], a discrete-time NCS model, based on the NCS model of e.g. [5], [6] is proposed, for time-varying delays $\tau_{k}$ that vary solely in the interval $(d-1) h \leq \tau_{k} \leq d h$, where $d$ is a constant integer, $h$ the sampling interval and $\tau_{k}$ the time-varying delay. As a consequence, time-delays that vary in an interval larger than the sampling interval, i.e. $\tau_{k} \in\left[\tau_{\min }, \tau_{\max }\right]$, with $\tau_{\max }-\tau_{\min }>h$ are not considered in [3], [4]. Note that this is a fundamental difference with arbitrary time-varying delays $\left(\tau_{\max }-\tau_{\min }>h\right)$, where message rejection can occur.

Discrete-time models that consider arbitrary time-varying delays that take values in an interval larger than the sampling

This work has been carried out as part of the Boderc project under the responsibility of the Embedded Systems Institute. This project is partially supported by the Dutch Ministry of Economic Affairs under the Senter TS program.

Marieke Cloosterman, Nathan van de Wouw, Maurice Heemels and Henk Nijmeijer are with the Department of Mechanical Engineering, Eindhoven University of Technology, The Netherlands \{m.b.g.cloosterman, n.v.d.wouw, w.p.m.h.heemels, h.nijmeijer\}@tue.nl interval are described in [7] and [8]. The model in [7] is based on [5] and describes the effects of multiple control updates during one sampling interval and vacancy sampling, where no new control input becomes available during the current sampling interval. The effect of message rejection, where more recent control data becomes available before the older data is implemented and therefore the older data is discarded, is, however, not considered in [7]. In [8] another stability analysis approach is described, without an explicit definition of the NCS model for large delays. For the analysis, the uncertain time-varying delays are divided in a constant and a time-varying part, where the time-varying part is allowed to be larger than the sampling interval. This results in a set of constant and uncertain matrices. Based on an overestimation of the uncertain matrices and a LyapunovKraskovskii approach for discrete-time systems, sufficient conditions for the stability analysis are proposed.

An alternative modeling approach is based on impulsive delay-differential equations, in which NCSs with variable sampling intervals, time-varying delays and packet dropouts are considered, see [9]. A main advantage of this modeling approach is the possibility to incorporate time-delays larger than the sampling interval without increasing model complexity, as is the case in the discrete-time modeling approach.

In this paper, we extend the model of [7], such that it includes message rejection. Due to this discrete-time representation, it is a different modeling approach than [9], which presents models in terms of continuous-time delay impulsive systems. Compared to the work in [8], we give an explicit definition of the uncertain functions, which depend on the uncertain time-varying delay, instead of an implicit one. We rewrite the proposed large-delay NCS model, using the real Jordan form of the continuous-time system matrices, such that we can separate constant and time-varying parameters, which removes conservativeness from the analysis. Based on this model, we propose LMIs that are sufficient for the stability analysis.

The paper is organized as follows. In Section II, we introduce the discrete-time NCS model for time-variations in the delay that are larger than one sampling interval. Section III discusses the stability analysis. In Section IV, simulation results illustrating the effectiveness of the proposed approach are presented. Finally, Section V gives the conclusions.

Notation: We denote the transpose of a matrix $A$ by $A^{T}$ and we write $P>0$ (or $P<0$ ) for a positive (or negative) definite matrix. With $*$ we denote the symmetric part of a matrix, with $\operatorname{dim}(J)$ the dimension of the square matrix $J$ and $0_{m, n}$ denotes a matrix of zeros of size $m \times n$. 


\section{NCS MODELS FOR LARGE DELAYS}

The NCS consists of a continuous-time plant, sampled with a constant sample-rate, and a discrete-time controller as is schematically depicted in Figure 1. In the model, both the computation time and the networked induced delays, i.e. the sensor-to-controller delay $\tau_{s c}$ and the controller-to-actuator delay $\tau_{c a}$, are incorporated. Under the assumption that the sensor acts in a time-driven fashion (i.e. sampling at the times $k h, k \in \mathbb{N}$ ), that the controller and actuator act in an event-driven fashion (i.e. responding instantaneously on newly arrived data) and that the controller is static and timeinvariant, all three delays can be combined into one single delay $\tau_{k}$ that delays the control input $u_{k}$ with respect to the corresponding measurement $y_{k}[10]$.

The continuous-time model of the NCS is given by:

$$
\begin{aligned}
& \dot{x}(t)=A x(t)+B u^{*}(t) \\
& u^{*}(t)=u_{k}, \text { for } t \in\left[k h+\tau_{k},(k+1) h+\tau_{k+1}\right],
\end{aligned}
$$

with $A \in \mathbb{R}^{n \times n}, B \in \mathbb{R}^{n \times m}$, the system matrices, $u^{*}(t) \in$ $\mathbb{R}^{m}$ the continuous-time control input if all inputs are implemented to the plant, i.e. all data at both the controller and actuator are received in a sequential order, which means that message rejection can not occur, $x(t) \in \mathbb{R}^{n}$ the state, $k h$ the sampling instants, $h$ the sampling-interval, $u_{k} \in \mathbb{R}^{m}$ the discrete-time control input calculated from the measurements at sampling instant $k h$ and $\tau_{k}$ the time-delay experienced by the output $y(k h)=x(k h)$ at sampling instant $k h$. At the end of this section, we will adapt the expression for $u^{*}(t)$, such that the effects of message rejection are included.

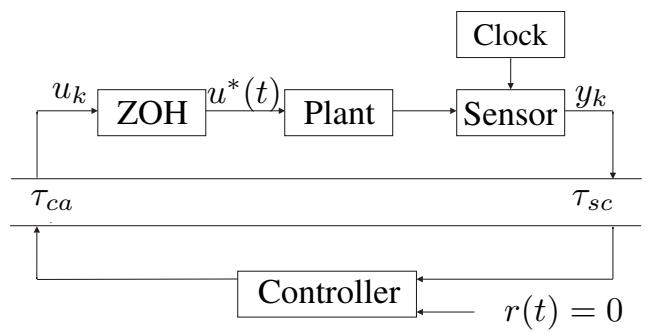

Fig. 1. Schematic overview of the networked control system.

To derive a discrete-time NCS model for time-varying delays that vary in an interval larger than the samplinginterval $\left(\tau_{k} \in\left[\tau_{\min }, \tau_{\max }\right]\right.$, with $\left.\tau_{\max }-\tau_{\min }>h\right)$, including message rejection, we use the standard, discretetime NCS model for constant large-delays, see e.g. [5]. This model is an exact discretization of (1), given by:

$$
\begin{aligned}
x_{k+1}= & e^{A h} x_{k}+\int_{0}^{h-\tau_{k-d+1}^{*}} e^{A s} d s B u_{k-d+1}+ \\
& \int_{h-\tau_{k-d+1}^{*}}^{h} e^{A s} d s B u_{k-d},
\end{aligned}
$$

with $d=\left\lceil\frac{\tau_{k}}{h}\right\rceil$, the smallest integer larger than or equal to $\frac{\tau_{k}}{h}$ and $\tau_{k}^{*}=\tau_{k}-(d-1) h$. This model is limited to constant time-delays or delays that vary at most within one sampling interval (i.e. $\left[\tau_{\min }, \tau_{\max }\right] \subseteq[(d-1) h, d h]$ for some constant integer $d$ ). This can be explained using Figure 2. In Figure 2, the delays are allowed to take values in $[0,2 h]$, which shows that the number of active control inputs in one sampling interval is variable and depends on the previous and current time-delay, which is not handled by (2).

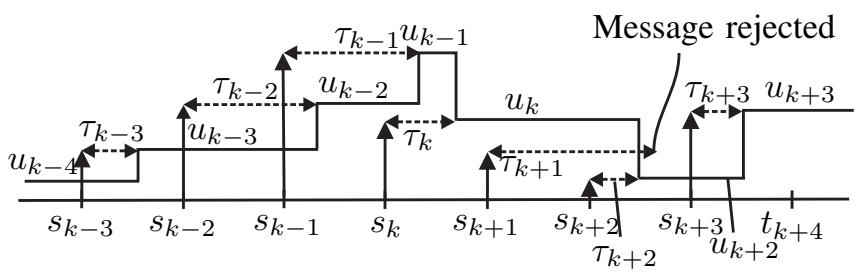

Fig. 2. Influence of time-delays that can be smaller and larger than the sampling interval (with e.g. $s_{k+1}:=(k+1) h$ and $\tau_{k} \in[0,2 h]$ ).

In the case that $\tau_{k} \in[0,2 h]$, the model that describes the NCS consists of the following subsystems:

$$
\begin{aligned}
& x_{k+1}= \\
& \left\{\begin{array}{c}
e^{A h} x_{k}+\int_{0}^{h-\tau_{k}} e^{A s} d s B u_{k}+\int_{h-\tau_{k}}^{h} e^{A s} d s B u_{k-1}, \\
\text { if } \tau_{k} \leq h \wedge \tau_{k-1} \leq h \\
e^{A h} x_{k}+\int_{0}^{h-\tau_{k-1}^{*}} e^{A s} d s B u_{k-1}+ \\
\int_{h-\tau_{k-1}^{h}}^{h} e^{A s} d s B u_{k-2}, \text { if } h \leq \tau_{k} \leq 2 h \wedge \\
h \leq \tau_{k-1} \leq 2 h \\
e^{A h} x_{k}+\int_{0}^{h} e^{A s} d s B u_{k-1}, \\
\text { if } h \leq \tau_{k} \leq 2 h \wedge \tau_{k-1} \leq h \\
e^{A h} x_{k}+\int_{0}^{h-\tau_{k}} e^{A s} d s B u_{k}+\int_{h-\tau_{k-1}^{*}}^{h} e^{A s} d s B u_{k-1} \\
+\int_{h-\tau_{k-1}^{*}}^{h} e^{A s} d s B u_{k-2}, \text { if } \tau_{k} \leq h \wedge \\
h \leq \tau_{k-1} \leq 2 h \wedge \tau_{k-1}-h<\tau_{k} \\
e^{A h} x_{k}+\int_{0}^{h-\tau_{k}} e^{A s} d s B u_{k}+\int_{h-\tau_{k}}^{h} e^{A s} d s B u_{k-2}, \\
\text { if } \tau_{k-1}-h \geq \tau_{k},
\end{array}\right.
\end{aligned}
$$

with $\tau_{k-1}^{*}=\tau_{k-1}-h$. The last subsystem describes the case where message rejection occurs, which results in loss of some control data $\left(u_{k-1}\right.$ in this case). Moreover, the second subsystem corresponds to (2) (with $d=2$ ). An NCS modeling approach that considers these different subsystems, except message rejection, is given in [7]. We will use their model description, which is based on (2), to develop an NCS model, including message rejection. In our model, during the sampling interval $[k h, k h+h)$, the control $u^{*}(t)$ takes values from the delayed inputs $u_{j}$ at the random instants $k h+t_{j}^{k}$, with $0 \leq t_{j}^{k} \leq h$. The integer $j$ satisfies $k-\bar{d} \leq j \leq k-\underline{d}$, with $\underline{d}=\left\lfloor\frac{\tau_{\min }}{h}\right\rfloor$, the largest integer smaller than or equal to $\frac{\tau_{\min }}{h}$ and $d=\left\lceil\frac{\tau_{\max }}{h}\right\rceil$ the smallest integer larger than or equal to $\frac{\tau_{\max }}{h}$. The time $k h+t_{j}^{k}$ depends on the time-delays and the values of $\underline{d}$ and $\bar{d}$, according to:

$$
\begin{aligned}
t_{j}^{k}=\min & {\left[\max \left\{0, \tau_{j}-(k-j) h\right\},\right.} \\
& \max \left\{0, \tau_{j+1}-(k-j-1) h\right\}, \ldots, \\
& \left.\max \left\{0, \tau_{k-\underline{d}}-\underline{d} h\right\}, h\right]
\end{aligned}
$$

with $j \in\{k-\bar{d}, k-\bar{d}+1, \ldots, k-\underline{d}\}$, the superscript ${ }^{k}$ indicating the sampling interval $[k h, k h+h), t_{j}^{k} \leq t_{j+1}^{k}$ and $0=t_{k-\bar{d}}^{k} \leq t_{k-\bar{d}+1}^{k} \leq \ldots \leq t_{k-\underline{d}}^{k} \leq t_{k-\underline{d}+1}^{k}:=h$.

Then, the NCS model for large delays becomes:

$$
x_{k+1}=e^{A h} x_{k}+\sum_{j=k-\bar{d}}^{k-\underline{d}} \int_{h-t_{j+1}^{k}}^{h-t_{j}^{k}} e^{A s} d s B u_{j}
$$


with $t_{k-d+1}^{k}:=h, t_{j}^{k}$ defined in (4) for $k-\bar{d} \leq j \leq k-\underline{d}$. This model contains all possible control inputs that can be active during the sampling interval $[k h, k h+h)$. Note that $t_{j}^{k}=t_{j+1}^{k}$ corresponds to the situation that the integral related to $u_{j}$ in (5) is zero and results in an inactive control input $u_{j}$ during the sampling interval $[k h, k h+h)$. This corresponds to the case of message rejection, vacancy sampling or a control input that has already been implemented in the previous sampling interval or will be implemented in the next sampling interval, due to the size of the time-delay.

Note that (5) is sufficient to model all the different situations that are given in (3), because each subsystem can be obtained by the definition of $t_{j}^{k}$ as in (4).

Remark Equation (5) was also stated in [7], however, without the explicit definition of $t_{j}^{k}$ as we presented in (4). Moreover, in [7] it is implicitly assumed that message rejection does not occur, as $t_{j}^{k}<t_{j+1}^{k}$ should hold for all $k-$ $\bar{d} \leq j \leq k-\underline{d}$. Finally, the model proposed here exhibits less uncertain parameters than that in [7], because we consider only $t_{j}^{k}$ as uncertain, time-varying parameters, while in [7] additional parameters are introduced that denote if a control input is active in the sampling interval $[k h, k h+h)$.

To make the model of (5) suitable for the stability analysis, we rewrite it in a state-space form with the state-vector $\xi_{k}=$ $\left(\begin{array}{lllll}x_{k}^{T} & u_{k-1}^{T} & u_{k-2}^{T} & \cdots & u_{k-\bar{d}}^{T}\end{array}\right)^{T}$. Then, the state-equation of the NCS model is given by:

$$
\begin{aligned}
& \xi_{k+1}=M\left(\tau_{k}\right) \xi_{k}+N\left(\tau_{k}\right) u_{k}, \\
& \text { with } M\left(\tau_{k}\right)=\left(\begin{array}{ccccc}
e^{A h} & \tilde{B}_{1} & \tilde{B}_{2} & \ldots & \tilde{B}_{\bar{d}} \\
0 & 0 & 0 & \ldots & 0 \\
0 & I & 0 & \ldots & 0 \\
\vdots & & \ddots & & \ldots \\
0 & \ldots & 0 & I & 0
\end{array}\right) \text {, } \\
& N\left(\tau_{k}\right)=\left(\begin{array}{lllll}
\tilde{B}_{0}^{T} & I & 0 & \ldots & 0
\end{array}\right)^{T} \text { and } \\
& \tilde{B}_{\rho}\left(\tau_{k}\right)=\int_{h-t_{k-\rho+1}^{k}}^{h-t_{k-\rho}^{k}} e^{A s} d s B
\end{aligned}
$$

for $\rho \in\{0,1, \ldots, \bar{d}\}$ and $t_{k-\rho}^{k}$ defined in (4), with $j=k-\rho$. Note that for $\rho<\underline{d}$, it holds that $\tilde{B}_{\rho}=0$.

Based on the insights obtained above, we can also adapt the control $u^{*}(t)$ in the continuous-time NCS model (1), such that it allows for message rejection of the current input $u_{k}$. Let us define $k^{*}(t):=\max \left\{k \in \mathbb{N} \mid k h+\tau_{k} \leq t\right\}$, which denotes the most recent control input available at time $t$. Then it holds for the NCS of (1) that

$$
u^{*}(t)=u_{k^{*}}(t),
$$

with $u^{*}(t)$ a piecewise constant signal. This is a kind of implicit description of the $\mathrm{ZOH}$-based control signal in the NCS. To follow the common representation of the control signal in the NCS literature, we make this more explicit in the following paragraph.

Let us define the additional integer parameter $v_{k}$ as the smallest integer, with $v_{k} \geq 1$, such that $u_{k+v_{k}}$ is a control input that is implemented after $k h+h$. In other words, if $u_{k}$ is implemented, $v_{k}$ denotes the next input $u_{k+v_{k}}$ that is implemented after $u_{k}$. If $u_{k}$ is rejected $v_{k}$ denotes the next input $u_{k+v_{k}}$ that is implemented after $u_{k-1}$, and so on if $u_{k-1}$ is rejected as well. The parameter $v_{k}$ is defined as:

$$
\begin{aligned}
& v_{k}= \\
& \begin{cases}1, & \text { if } \tau_{k+1}-h<\tau_{k+2} \wedge \tau_{k+1}-2 h<\tau_{k+3} \wedge \\
& \ldots \wedge \tau_{k+1}-\bar{d} h<\tau_{k+\bar{d}+1} \\
2, & \text { if } \tau_{k+1}-h \geq \tau_{k+2} \wedge \tau_{k+2}-h<\tau_{k+3} \wedge \\
& \cdots \wedge \tau_{k+2}-(\bar{d}-1) h<\tau_{k+\bar{d}+1} \\
\vdots & \\
\bar{d}, \quad & \text { if } \tau_{k+1}-(\bar{d}-1) h \geq \tau_{k+\bar{d}} \wedge \\
& \tau_{k+2}-(\bar{d}-2) h \geq \tau \\
& \tau_{k+\bar{d}-1}-h \geq \tau_{k+\bar{d}} \wedge \tau_{k+\bar{d}} \wedge h<\tau_{k+\bar{d}+1} \\
\bar{d}+1, & \text { if } \tau_{k+1}-\bar{d} h \geq \tau_{k+\bar{d}+1} \wedge \\
& \tau_{k+2}-(\bar{d}-1) h \geq \tau_{k+\bar{d}+1} \wedge \ldots \wedge \\
& \tau_{k+\bar{d}-1}-2 h \geq \tau_{k+\bar{d}+1} \wedge \tau_{k+\bar{d}}-h=\tau_{k+\bar{d}+1}\end{cases}
\end{aligned}
$$

Based on this definition of $v_{k}$, the continuous-time NCS model that incorporates delays larger than the sampling interval and message rejection becomes, for known values of $v_{k}$ :

$$
\begin{aligned}
& \dot{x}(t)=A x(t)+B u^{*}(t) \\
& u^{*}(t)= \\
& \begin{cases}u_{k}, & \text { for } t \in\left[k h+\tau_{k},\left(k+v_{k}\right) h+\tau_{k+v_{k}}\right] \\
& \wedge \tau_{k}-v_{k} h<\tau_{k+v_{k}} \wedge 1 \leq v_{k} \leq \bar{d}+1 \\
u_{k-1}, & \text { for } t \in\left[(k-1) h+\tau_{k-1},\left(k+v_{k}\right) h+\tau_{k+v_{k}}\right] \\
& \wedge \tau_{k}-v_{k} h \geq \tau_{k+v_{k}} \\
& \wedge \tau_{k-1}-\left(v_{k}+1\right) h<\tau_{k+v_{k}} \wedge 1 \leq v_{k} \leq \bar{d}\end{cases} \\
& \left\{\begin{array}{l}
\vdots \\
u_{k-\bar{d}+1}, \quad \text { for } t \in\left[(k-\bar{d}+1) h+\tau_{k-\bar{d}+1},\left(k+v_{k}\right) h+\tau_{k+v_{k}}\right]
\end{array}\right. \\
& \left\{\begin{aligned}
& \wedge \tau_{k}-v_{k} h \geq \tau_{k+v_{k}} \wedge \\
& \tau_{k-1}-\left(v_{k}+1\right) h \geq \tau_{k+v_{k}} \wedge \ldots \wedge
\end{aligned}\right. \\
& \tau_{k-\bar{d}+2}-(v+\bar{d}-2) h \geq \tau_{k+v_{k}} \wedge \\
& \tau_{k-\bar{d}+1}-\left(v_{k}+\bar{d}-1\right) h<\tau_{k+v_{k}} \wedge 1 \leq v_{k} \leq 2 \\
& u_{k-\bar{d}}, \quad \text { for } t \in\left[(k-\bar{d}) h+\tau_{k-\bar{d}},\left(k+v_{k}\right) h+\tau_{k+v_{k}}\right] \wedge \\
& \tau_{k}-v_{k} h \geq \tau_{k+v_{k}} \wedge \\
& \tau_{k-1}-\left(v_{k}+1\right) h \geq \tau_{k+v_{k}} \wedge \ldots \wedge \\
& \tau_{k-\bar{d}+1}-\left(v_{k}+\bar{d}-1\right) h \geq \tau_{k+v_{k}} \wedge v_{k}=1,
\end{aligned}
$$

with $k=\left\lfloor\frac{t}{h}\right\rfloor$. Note that in the last subsystem in (10), $\tau_{k-\bar{d}+1}-\left(v_{k}+\bar{d}-1\right) h>\tau_{k+v_{k}}$ will never occur, but $\tau_{k-\bar{d}+1}-\left(v_{k}+\bar{d}-1\right) h=\tau_{k+v_{k}}$ is still possible if $\tau_{\max }=\bar{d}$.

\section{STABILITY OF NCSS WITH LARGE DELAYS}

In this section, we propose techniques for the stability analysis of the NCS model described in Section II. To do so, we first define a different formulation of the NCS model of (6). To solve the integrals in the matrices $\tilde{B}_{\rho}$ in (7) in a general way, the real Jordan form [11], [12] of the continuous-time system matrix $A$ is used, which is given by:

$$
A=Q J Q^{-1}
$$

with $J$ the real Jordan form, representing the eigenvalues and $Q$ a matrix that contains the generalized eigenvectors. Suppose that $A$ has $p$ distinct eigenvalues $\lambda_{\tilde{i}}$, with $J_{\tilde{i}, \tilde{j}}$ the corresponding Jordan blocks for the eigenvalue $\lambda_{\tilde{i}}$ and $\tilde{j}=\left\{1,2, \ldots, g_{\tilde{i}}\right\}$, with $g_{\tilde{i}}$ the geometric multiplicity of the 
$\tilde{i}^{t h}$ eigenvalue, with $\tilde{i}=1,2, \ldots, p$. Then, it holds, for real eigenvalues $\lambda_{\tilde{i}}$, that:

$$
e^{J s}=\sum_{\tilde{i}=1}^{p} \sum_{\breve{j}=0}^{a_{\tilde{i}}-1} \frac{s^{\breve{j}}}{\breve{j} !} e^{\lambda_{\tilde{i}} s} S_{\tilde{i}, \breve{j}},
$$

with $S_{\tilde{i}, \breve{j}}$ an appropriate matrix of the same dimension as $J$, with a one at the matrix entries corresponding to $\frac{s^{\breve{j}}}{j !} e^{\lambda_{\tilde{i}} s}$ and a zero at all other matrix entries and $a_{\tilde{i}} \in \mathbb{Z}^{+}$the size of the largest Jordan block that corresponds to $\lambda_{\tilde{i}}$, i.e.

$$
a_{\tilde{i}}=\max _{\tilde{j} \in\left\{1,2, \ldots, g_{\tilde{i}}\right\}}\left(\operatorname{dim} J_{\tilde{i}, \tilde{j}}\right) .
$$

Similar expressions can be obtained for complex eigenvalues.

Rewriting (6), (7), using (11) and (12), leads to the following general NCS representation:

$$
\xi_{k+1}=\left(F_{0}+\sum_{i=1}^{\beta} \alpha_{i} F_{i}\right) \xi_{k}+\left(G_{0}+\sum_{i=1}^{\beta} \alpha_{i} G_{i}\right) u_{k},
$$

with $F_{i}$ and $G_{i}$ constant matrices that are obtained by solving the integrals in (6), (7) with $A$ replaced by (11) and $\alpha_{i}$, $i \in\{1,2, \ldots, \beta\}$, a time-varying parameter that depends on the eigenvalue $\lambda_{\tilde{i}}, h$ and one of the time-varying delays introduced through $t_{j}^{k}$ in (4). The parameter $\beta$ denotes the number of time-varying parameters in the sampling interval $[k h, k h+h):$

$$
\beta=(\bar{d}-\underline{d}) \nu,
$$

with $\nu$ the number of parameters (from (12)) that depend on the eigenvalues of $A$ :

$$
\nu=\sum_{\tilde{i}=1}^{p} a_{\tilde{i}},
$$

with $\nu$ always smaller than or equal to $n$. As an illustration, the matrices $F_{i}$ and $G_{i}, i=\{0,1, \ldots, \beta\}$, are given for the case with all eigenvalues equal to zero in Appendix II.

\section{A. Stability Analysis}

To derive stability conditions for the discrete-time NCS model (6), we adopt the control law

$$
u_{k}=-K \xi_{k}
$$

with $K \in \mathbb{R}^{m \times(\bar{d} m+n)}$. Note that the state-feedback control law $u_{k}=-\bar{K} x_{k}$, is a special case of (17), with $K=$ $\left(\begin{array}{ll}\bar{K} & 0\end{array}\right), \bar{K} \in \mathbb{R}^{m \times n}$ and 0 a matrix with zeros. System (14), with control law (17) contains the uncertain time-varying parameters $\alpha_{i}$, which may be nonlinear in the parameter $t_{j}^{k}$. These parameters form, together with the constant matrices $F_{i}$ and $G_{i}, i=\{0,1, \ldots, \beta\}$, sets of matrices that describe all possible system equations of (6):

$$
\begin{aligned}
& \mathcal{F}=\left\{F_{0}+\sum_{i=1}^{\beta} \alpha_{i}\left(t_{j}^{k}\right) F_{i}: t_{j}^{k} \in\left[t_{j, \text { min }}^{k}, t_{j, \text { max }}^{k}\right]\right\} \\
& \mathcal{G}=\left\{G_{0}+\sum_{i=1}^{\beta} \alpha_{i}\left(t_{j}^{k}\right) G_{i}: t_{j}^{k} \in\left[t_{j, \text { min }}^{k}, t_{j, \text { max }}^{k}\right]\right\} .
\end{aligned}
$$

To determine the minimum and maximum values of the uncertain parameters $\alpha_{i}$ in (18), we define the minimum and maximum values of $t_{j}^{k}$, dependent of the size of the timedelays. We define the minimum value of $t_{j}^{k}$ as:

$$
t_{j, \min }^{k}= \begin{cases}\tau_{\min }-\underline{d} h & \text { if } j=k-\underline{d} \\ 0 & \text { if } j<k-\underline{d},\end{cases}
$$

and the maximum value of $t_{j}^{k}$ as:

$$
t_{j, \text { max }}^{k}= \begin{cases}h & \text { if } j>k-\bar{d}+1 \\ \tau_{\text {max }}-(\bar{d}-1) h & \text { if } j=k-\bar{d}+1 .\end{cases}
$$

To guarantee the stability of the equilibrium point $\xi=0$ of system (14), with the uncertain parameters $t_{j}^{k}$, defined in (4), we will use a common quadratic Lyapunov function $V\left(\xi_{k}\right)=\xi_{k}^{T} P \xi_{k}$. Consequently, the following LMI condition is sufficient:

$$
\begin{aligned}
P=P^{T} & >0 \\
C(\tau)^{T} P C(\tau)-P & <-\gamma P, \forall \tau \in\left[\tau_{\text {min }}, \tau_{\text {max }}\right],
\end{aligned}
$$

with $C(\tau)=F_{0}-G_{0} K+\sum_{i=1}^{\beta} \alpha_{i}\left(F_{i}-G_{i} K\right)$ and $0 \leq$ $\gamma<1$. This is, based on the Schur complement, equal to:

$$
\left(\begin{array}{cc}
(1-\gamma) P & * \\
P\left(F_{0}-G_{0} K+\sum_{i=1}^{\beta} \alpha_{i}\left(F_{i}-G_{i} K\right)\right. & P
\end{array}\right)>0 .
$$

Obviously, (22) represents an infinite number of LMIs. In Theorem 1, we will propose a stability condition for $\tau_{k} \in$ $\left[\tau_{\min }, \tau_{\max }\right]$, with $0 \leq \tau_{\min } \leq \tau_{\max }$, based on a finite number of LMIs.

Theorem 1: Consider the NCS of (6), with a known controller (17) and time-varying delays taken from a bounded set $\tau_{k} \in\left[\tau_{\min }, \tau_{\max }\right], 0 \leq \tau_{\min } \leq \tau_{\max }$. Define the sets of matrices $\mathcal{H}_{\mathcal{F}}$ and $\mathcal{H}_{\mathcal{G}}$ :

$$
\begin{aligned}
& \mathcal{H}_{\mathcal{F}}=\left\{\bar{F}_{0}+\sum_{i=1}^{\beta} \delta_{i} \bar{F}_{i}: \delta_{i} \in\{0,1\}, i=\{1,2, \ldots, \beta\}\right\}, \\
& \mathcal{H}_{\mathcal{G}}=\left\{\bar{G}_{0}+\sum_{i=1}^{\beta} \delta_{i} \bar{G}_{i}: \delta_{i} \in\{0,1\}, i=\{1,2, \ldots, \beta\}\right\},
\end{aligned}
$$

with $\bar{F}_{0}=F_{0}+\sum_{i=1}^{\beta} \underline{\alpha}_{i} F_{i}, \bar{F}_{i}=\left(\bar{\alpha}_{i}-\underline{\alpha}_{i}\right) F_{i}, \bar{G}_{0}=G_{0}+$ $\sum_{i=1}^{\beta} \underline{\alpha}_{i} G_{i}, \bar{G}_{i}=\left(\bar{\alpha}_{i}-\underline{\alpha}_{i}\right) G_{i}$, and

$$
\begin{aligned}
& \bar{\alpha}_{i}=\max _{t_{j}^{k} \in\left[t_{j, \text { min }}^{k}, t_{j, \text { max }}^{k}\right]} \alpha_{i}\left(t_{j}^{k}\right), \\
& \underline{\alpha}_{i}=\min _{t_{j}^{k} \in\left[t_{j, \text { min }}^{k}, t_{j, \text { max }}^{k}\right]} \alpha_{i}\left(t_{j}^{k}\right),
\end{aligned}
$$

the maximum and minimum value of $\alpha_{i}$, respectively, with $t_{j, \min }^{k}$ and $t_{j, \text { max }}^{k}$ defined in (19) and (20), respectively.

If there exist a matrix $P \in \mathbb{R}^{(n+\bar{d} m) \times(n+\bar{d} m)}$ and a scalar $0 \leq \gamma<1$, such that the following LMI conditions are satisfied:

$$
\left.\begin{array}{cc}
(1-\gamma) P & \left(H_{F, s}-H_{G, s} K\right)^{T} P \\
P\left(H_{F, s}-H_{G, s} K\right) & P
\end{array}\right)>0,
$$

and $s=\left\{1,2, \ldots, 2^{\beta}\right\}$, then (10), (17) is GAS for any sequence of time-varying delays $\tau \in\left[\tau_{\min }, \tau_{\max }\right]$.

Proof: The proof is given in Appendix I.

These LMIs contain only stability analysis results. Note that performance measures can be included in these LMIs as well. In [13], the LMIs are augmented such that a certain ISS bound can be guaranteed in the face of disturbances. 


\section{ILLUSTRATIVE EXAMPLE}

In this section, we will apply the proposed results to a second-order motion control example, obtained from the document printing domain, as in [14]. We limit ourselves to one single motor driving one roller pair that is used to transport a sheet through part of the printer paperpath. The continuous-time motor-roller model is given by:

$$
\begin{aligned}
\dot{x} & =A x+B u \\
y & =C x,
\end{aligned}
$$

with $A=\left(\begin{array}{ll}0 & 1 \\ 0 & 0\end{array}\right), B=\left(\begin{array}{c}0 \\ \frac{n r_{R}}{J_{M}+n^{2} J_{R}}\end{array}\right), C=\left(\begin{array}{ll}1 & 0 \\ 0 & 1\end{array}\right)$ and $x=\left(\begin{array}{ll}x_{s}^{T} & \dot{x}_{s}^{T}\end{array}\right)$ the state vector, which contains the sheet position and velocity. Moreover, $J_{M}=1.95 \cdot 10^{-5} \mathrm{kgm}^{2}$ the inertia of the motor, $J_{R}=6.5 \cdot 10^{-5} \mathrm{kgm}^{2}$ the inertia of the roller, $r_{R}=14 \cdot 10^{-3} \mathrm{~m}$ the radius of the roller, $n=0.2$ the transmission ratio between motor and roller and $u$ the motor torque. We assume that the sampling interval $h=1 \mathrm{~ms}$ is constant and that the state-feedback controller is given by $u_{k}=-\bar{K} x_{k}$, with $\bar{K}=\left(\begin{array}{ll}K_{1} & K_{2}\end{array}\right)$ and a constant position controller gain $K_{1}=50$. We determine the controller gains $K_{2}$ that still stabilize the system with timevarying delays $\tau_{k} \in\left[0, \tau_{\max }\right]$, with $\tau_{\max } \leq 2 h$, according to Theorem 1 with $\gamma=0$. The maximum and minimum allowable controller gains $K_{2}$ are given by the solid line in Figure 3. Note that for delays larger than the sampling interval $h$, the possibility of message rejection is included, as given in (5), (4). Compared to the previously published stability conditions in [14], which are depicted by the dotted line in Figure 3, the results obtained by Theorem 1 are clearly less conservative, due to the usage of the Jordan form representation of (14) of the NCS, which results in a reduction of the number of LMIs and a much tighter overapproximation of $F_{i}$ and $G_{i}$ than in [14]. The dashed line in Figure 3 gives the values of a periodic delay sequence with two delays $\left(\tau_{a}, \tau_{b}, \tau_{a}, \tau_{b}, \ldots\right)$, with $\tau_{a}=0.2 \mathrm{~ms}$ and $\tau_{b}=0.6$ $\mathrm{ms}(\bar{K}=[5011.8], h=1 \mathrm{~ms})$, that yields an unstable closedloop system. However, for constant delays (i.e. only $\tau_{a}$ or only $\tau_{b}$ ) the corresponding system is stable (see for more details [14]). For comparison, the minimum and maximum allowable controller gains for constant time-delays equal to $\tau_{\max }$ are depicted by the dash-dotted line in Figure 3. Such examples underline the need fot stability results for timevarying delays. We remark that the difference between the lines for constant and time-varying delays is similar for other values of $K_{1}$. This difference reveals the fact that the stability bound is hardly conservative.

\section{CONCLUSIONS AND FUTURE WORKS}

In this paper we extended the NCS model for large delays of [7] to incorporate the effect of message rejection. Moreover, we introduced an explicit definition of this extended discrete-time NCS model. For this NCS model, sufficient LMI conditions for stability analysis are proposed. By using the real Jordan form of the original continuous-time plant, the number of uncertain parameters is much smaller than in

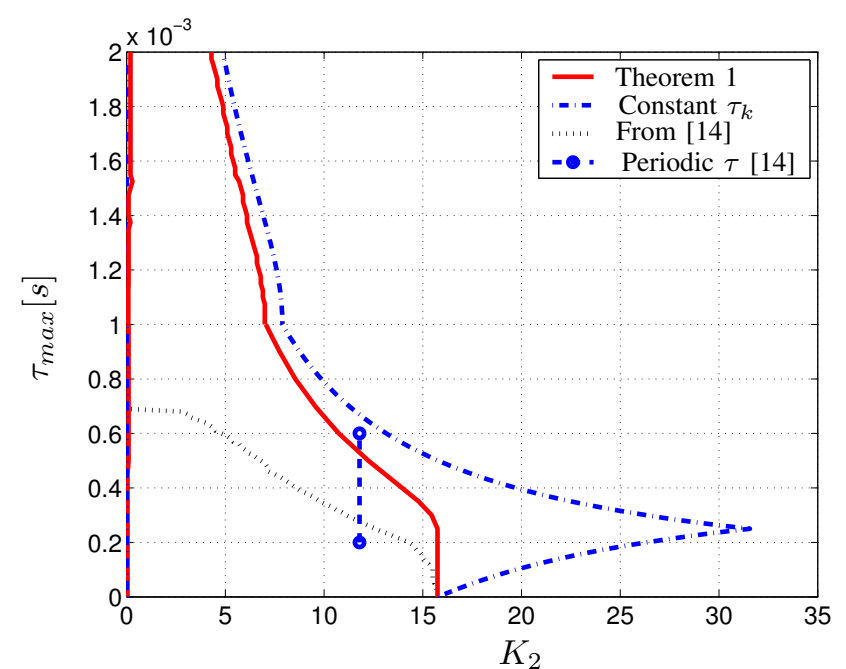

Fig. 3. Minimum and maximum allowable controller gains $K_{2}$, for $h=1 \mathrm{~ms}, K_{1}=50$ and time-varying delays in the interval $\left[0, \tau_{\max }\right]$ for Theorem 1 and based on [14], and for constant delays equal to $\tau_{\max }$.

[14], thereby resulting in a reduction of the number of LMIs and the conservatism, as illustrated by the example.

Future work will deal with a model extension including that packet dropouts.

\section{APPENDIX I \\ PROOF OF THEOREM 1}

The proof of Theorem 1 consists of three parts. First, we exploit a convex overapproximation of the sets $\mathcal{F}$ and $\mathcal{G}$, defined in (18). Second, we prove the stability of the discrete-time NCS model, based on the common quadratic Lyapunov function $V\left(\xi_{k}\right)=\xi_{k}^{T} P \xi_{k}$. Third, we investigate the intersample behavior to prove stability of the continuoustime NCS model (10), (17). For the time-varying parameter $\alpha_{i}$, given in (24), we can write any $\alpha_{i} \in\left[\underline{\alpha}_{i}, \bar{\alpha}_{i}\right]$ as $\alpha_{i}=$ $\underline{\alpha}_{i}+\delta_{i}\left(\bar{\alpha}_{i}-\underline{\alpha}_{i}\right)$, for some $\delta_{i} \in[0,1]$ and $i=\{1,2, \ldots, \beta\}$. Hence, the sets $\overline{\mathcal{F}}$ and $\overline{\mathcal{G}}$, are defined as:

$$
\begin{aligned}
& \overline{\mathcal{F}}=\left\{\bar{F}_{0}+\sum_{i=1}^{\beta} \delta_{i} \bar{F}_{i}: \delta_{i} \in[0,1], i=\{1,2, \ldots, \beta\}\right\}, \\
& \overline{\mathcal{G}}=\left\{\bar{G}_{0}+\sum_{i=1}^{\beta} \delta_{i} \bar{G}_{i}: \delta_{i} \in[0,1], i=\{1,2, \ldots, \beta\}\right\},
\end{aligned}
$$

with $\bar{F}_{0}=F_{0}+\sum_{i=1}^{\beta} \underline{\alpha}_{i} F_{i}, \bar{F}_{i}=\left(\bar{\alpha}_{i}-\underline{\alpha}_{i}\right) F_{i}, \bar{G}_{0}=G_{0}+$ $\sum_{i=1}^{\beta} \underline{\alpha}_{i} G_{i}, \bar{G}_{i}=\left(\bar{\alpha}_{i}-\underline{\alpha}_{i}\right) G_{i}$. This is an overapproximation of the set $\mathcal{F}$ and $\mathcal{G}$ in the sense that $\mathcal{F} \subseteq \overline{\mathcal{F}}$ and $\mathcal{G} \subseteq \overline{\mathcal{G}}$, respectively. Due to the definition of the new uncertainty parameters $\delta_{i}$, the sets $\overline{\mathcal{F}}$ and $\overline{\mathcal{G}}$ are still infinite. However, each matrix in this set can be written as a convex combination of the generators of this set, which are defined as the set $\mathcal{H}_{\mathcal{F}}$ and $\mathcal{H}_{\mathcal{G}}$ in (23). Note that the set $\mathcal{H}_{\mathcal{F}}$ and $\mathcal{H}_{\mathcal{G}}$ consist of $2^{\beta}$ matrices, which we denote individually by $H_{F, s}, H_{G, s}, s=\left\{1,2, \ldots, 2^{\beta}\right\}$. Based on these generators, a convex overapproximation of $\overline{\mathcal{F}}$ and $\overline{\mathcal{G}}$ can be given by:

$$
\begin{aligned}
& \operatorname{co}\left(\mathcal{H}_{\mathcal{F}}\right)=\left\{\sum_{s=1}^{2^{\beta}}\left(\mu_{s} H_{F, s}\right): \sum_{s=1}^{2^{\beta}} \mu_{s}=1, \mu_{s} \in[0,1],\right. \\
& \left.\quad s=\left\{1,2, \ldots, 2^{\beta}\right\}\right\}, \\
& \operatorname{co}\left(\mathcal{H}_{\mathcal{G}}\right)=\left\{\sum_{s=1}^{2^{\beta}}\left(\mu_{s} H_{G, s}\right): \sum_{s=1}^{2^{\beta}} \mu_{s}=1, \mu_{s} \in[0,1],\right. \\
& \left.\quad s=\left\{1,2, \ldots, 2^{\beta}\right\}\right\},
\end{aligned}
$$


in the sense that

$$
\begin{aligned}
& \mathcal{F} \subseteq \overline{\mathcal{F}} \subseteq \operatorname{co}\left(\mathcal{H}_{\mathcal{F}}\right), \\
& \mathcal{G} \subseteq \overline{\mathcal{G}} \subseteq \operatorname{co}\left(\mathcal{H}_{\mathcal{G}}\right) .
\end{aligned}
$$

Next, we show that indeed (25) is sufficient to guarantee the satisfaction of (22). Since (25) holds for all $H_{F} \in \mathcal{H}_{\mathcal{F}}$ and $H_{G} \in \mathcal{H}_{\mathcal{G}}$, for any $\mu_{s} \geq 0$ and $\sum_{s=1}^{2^{\beta}} \mu_{s}=1$, (23) implies

$$
\begin{aligned}
0 & <\sum_{s=1}^{2^{\beta}} \mu_{s}\left(\begin{array}{cc}
(1-\gamma) P & * \\
P\left(H_{F, s}-H_{G, s} K\right) & P
\end{array}\right) \\
& =\left(\begin{array}{cc}
(1-\gamma) P & * \\
\sum_{s=1}^{2^{\beta}} \mu_{s} P\left(H_{F, s}-H_{G, s} K\right) & P
\end{array}\right) .
\end{aligned}
$$

Consequently, it holds that:

$$
\left(\begin{array}{cc}
(1-\gamma) P & * \\
P\left(\overline{H_{F, s}}-\overline{H_{G, s}} K\right) & P
\end{array}\right)>0,
$$

$\forall \overline{H_{F, s}} \in \operatorname{co}\left(\mathcal{H}_{\mathcal{F}}\right)$ and $\forall \overline{H_{G, s}} \in \operatorname{co}\left(\mathcal{H}_{\mathcal{G}}\right), s \in\left\{1,2, \ldots, 2^{\beta}\right\}$. Based on (27), we have that (29) implies (22), which is, based on the Schur complement, equal to (21). This shows that $V\left(\xi_{k}\right)=\xi_{k}^{T} P \xi_{k}$ is a common quadratic Lyapunov function for (14), (17) which proves GAS of the origin $\xi=0$ of (14), (17) and by equivalence of (6), (17).

With a similar reasoning as in [14], it can be proven that the intersample behavior (and therefore the continuous-time system) is GAS if the NCS model is GAS, i.e. the LMIs (25) are feasible. This proves GAS of $x=0$ of (10), (17).

\section{APPENDIX II \\ JORDAN FORMS OF THE LARGE DELAY NCS MODEL}

For the case with all eigenvalues equal to zero the parameter $\alpha_{i}$ in (14) is defined as:

$$
\alpha_{i}=\left\{\begin{array}{l}
\frac{\left(h-t_{k-\underline{d}}^{k}\right)^{\breve{j}}}{\breve{j} !}, \quad \text { for } \breve{j}=i-1 \wedge i \in\{1,2, \ldots, \nu\}, \\
\frac{\left(h-t_{k-\underline{d}+1}^{k}\right)^{\breve{j}}}{\breve{j} !}, \quad \text { for } \breve{j}=i-\nu-1 \wedge \\
i \in\{\nu+1, \nu+2, \ldots, 2 \nu\}, \\
\frac{\left(h-t_{k-\bar{d}+1}^{k}\right)^{\breve{j}}}{\breve{j} !}, \quad \text { for } \breve{j}=i-(\beta-\nu+1) \wedge \\
i \in\{\beta-\nu+1, \ldots, \beta\} .
\end{array}\right.
$$

The corresponding matrices are given by:

$$
F_{0}=\left(\begin{array}{ccccc}
\Theta_{1} & 0_{n, m} & 0_{n, m} & \ldots & \Theta_{2} \\
0 & 0 & 0 & \ldots & 0 \\
0 & I & 0 & \ldots & 0 \\
\vdots & & \ddots & & \vdots \\
0 & \ldots & 0 & I & 0
\end{array}\right), F_{i}=\left(\begin{array}{c}
\Psi_{i} \\
0_{\bar{d} m, n+\bar{d} m}
\end{array}\right),
$$

with $\Theta_{1}=Q e^{J h} Q^{-1}, \Theta_{2}=Q \sum_{\breve{j}=0}^{a_{\tilde{i}}-1} \frac{h^{\breve{j}+1}}{(\breve{j+1}) j !} S_{\tilde{i}, \breve{j}} Q^{-1} B$ and

$$
G_{0}=\left(\begin{array}{c}
0_{n, m} \\
I_{m, m} \\
0_{(\bar{d}-1) m, m}
\end{array}\right), G_{i}=\left(\begin{array}{c}
\Xi \\
0_{\bar{d} m, m}
\end{array}\right) .
$$

Before we give $\Psi_{i}$ and $\Xi_{i}$, let us first define $\Upsilon_{i}=$ $Q T_{i} Q^{-1} B, \tilde{s}_{1}=(\bar{d}-\underline{d}-1) m, \tilde{s}_{2}=\tilde{s}_{1}-m$ and $\tilde{s}_{2}=\tilde{s}_{2}-m$. Then, it holds that

$$
\Xi_{i}= \begin{cases}\Upsilon_{i}, & \text { if } i \in\{1,2, \ldots, \nu\} \wedge \underline{d}=0 \\ 0_{n, m}, & \text { if } i>\nu \wedge \underline{d}=0 \text { or } \underline{d}>0,\end{cases}
$$

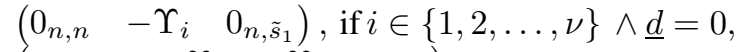

$$
\begin{aligned}
& \left(\begin{array}{llll}
0_{n, n+\underline{d} m} & \Upsilon_{i} & -\Upsilon_{i} & 0_{n, \tilde{s}_{2}}
\end{array}\right), \\
& \text { if } i \in\{\nu+1, \nu+2, \ldots, 2 \nu\} \wedge \underline{d}=0 \text {, } \\
& \text { or } i \in\{1,2, \ldots, \nu\} \wedge \underline{d}>0 \text {, } \\
& \Psi_{i}=\left\{\begin{array}{c}
\left(0_{n, n+(\underline{d}+1) m} \Upsilon_{i}-\Upsilon_{i} \quad 0_{n, \tilde{s}_{3}}\right), \\
\quad \text { if } i \in\{2 \nu+1,2 \nu+2, \ldots, 3 \nu\} \wedge \underline{d}=0
\end{array}\right. \\
& \text { or } i \in\{\nu+1, \nu+2, \ldots, 2 \nu\} \wedge \underline{d}>0 \text {, } \\
& \left(0_{n, n+(\bar{d}-2) m} \Upsilon_{i}-\Upsilon_{i}\right), \\
& \text { if } i \in\{(\bar{d}-\underline{d}-1) \nu+1, \ldots, \beta\}
\end{aligned}
$$

and for $\underline{d}>0$ it holds that $\Xi_{i}=0_{n, m} \forall i \in\{1,2, \ldots, \beta\}$. Moreover, it holds that:

$$
\begin{aligned}
& T_{i}= \\
& \left\{\begin{array}{c}
S_{1, \breve{j}} \text { for } \breve{j}=i-1 \wedge i=\{1,2, \ldots, \nu\}, \\
S_{1, \breve{j}} \text { for } \breve{j}=i-\nu-1 \wedge i=\{\nu+1, \nu+2, \ldots, 2 \nu\}, \\
\vdots \\
S_{1, \breve{j}} \text { for } \breve{j}=i-\beta+\nu-1 \wedge i=\{\beta-\nu+1, \ldots, \beta\} .
\end{array}\right.
\end{aligned}
$$

[1] Y. Tipsuwan and M.-Y. Chow, "Control methodologies in networked control systems," Control Engineering Practice, vol. 11, pp. 10991111,2003

[2] J. P. Hespanha, P. Naghshtabrizi, and Y. Xu, "A survey of recent results in networked control systems," Proc. of the IEEE, pp. 138162, January 2007.

[3] Y. Yang and Y.-J. Wang, "Modeling and control for NCS with timevarying long delays," in Proc. of the 4th Int. Conf. on Machine Learning and Cybernetics, Guangzhou, August 2005, pp. 1407-1411.

[4] S. Li, Z. Wang, and Y. Sun, "Observer-based compensator design for networked control systems with long time delays," in Proc. of the IEEE Industrial Electronics Society, Busan, Korea, November 2004, pp. 678-683.

[5] K. J. Åström and B. Wittenmark, Computer controlled systems, theory and design. Englewood Cliffs: Prentice-Hall International, Inc, 1990.

[6] B. W. Zhang, M. S. Branicky, and S. M. Phillips, "Stability of networked control systems," IEEE Control Systems Magazine, vol. 21, no. 1, pp. 84-99, February 2001.

[7] Y. Yang, D. Xu, M. Tan, and X. Dai, "Stochastic stability analysis and control of networked control systems with randomly varying long time-delays," in Proc. of the 5th World Congress on Intelligent Control and Automation, Hangzhou, China, June 2004, pp. 1391-1395.

[8] Y.-J. Pan, H. J. Marquez, and T. Chen, "Stabilization of remote control systems with unknown time varying delays by LMI techniques," Int. Journal of Control, vol. 79, no. 7, pp. 752-763, July 2006.

[9] P. Naghshtabrizi and J. P. Hespanha, "Stability of network control systems with variable sampling and delays," in Proc. of the Forty-Fourth Annual Allerton Conf. on Communication, Control, and Computing, A. Singer and C. Hadjicostis, Eds., Sept. 2006.

[10] J. Nilsson, Real-Time Control Systems with Delays. Lund, Sweden: $\mathrm{PhD}$ thesis, Department of Automatic Control, Lund Institute of Technology, 1998.

[11] M. W. Hirsch and S. Smale, Differential Equations, Dynamical Systems and Linear Algebra. New York: Pure and Applied Mathematics 60, Academic Press, 1974.

[12] C. Moler and C. van Loan, "Nineteen dubious ways to compute the exponential of a matrix," SIAM review, vol. 20, pp. 801-836, 1978.

[13] N. van de Wouw, P. Naghshtabrizi, M. Cloosterman, and J. P. Hespanha, "Tracking control for networked control systems," in Proc. of the 46th Conf. on Decision and Control, New Orleans, USA, 2007.

[14] M. Cloosterman, N. van de Wouw, M. Heemels, and H. Nijmeijer, "Robust stability of networked control systems with time-varying network-induced delays," in Proc. of the 45th Conf. on Decision and Control, San Diego, USA, December 2006, pp. 4980-4985. 